\title{
ENSAYO
}

\section{La Productividad Científica DE Economía y Administración en ChILE. Un AnÁlisis Comparativo*}

\author{
Claudia Contreras \\ CONICYT \\ GONZALO EDWARDS \\ Pontificia Universidad Católica de Chile \\ Alejandra Mizala \\ Universidad de Chile
}

The first part of this paper compares the volume and scientific productivity of Business Administration and Economics in Chile with the rest of scientific disciplines at the national and international levels. Given that scientific productivity is heterogeneous among different disciplines, the comparisons utilize an indicator that measures their impact relative to the world in the same field. The second part of the paper compares the amounts of public resources allocated to research in the different fields and discusses their relative efficiency in Chile.

\section{JEL: A 12}

Keywords: Business Administration and Economics, Scientific Productivity, Citations, Impact Factor, Impact Relative to Field.

\section{INTRODUCCIÓN}

El objetivo de este trabajo es doble; por una parte, nos interesa contrastar el volumen y la calidad de la productividad científica de las ciencias económicas y administrativas en Chile con el resto de las disciplinas científicas a nivel nacio-

\footnotetext{
*Agradecemos la ayuda de Carla Benedetti, la colaboración de María Elena Boisier y Macarena Verdugo y los comentarios de Eugenio Bobenrieth, Alan Farcas, Ronald Fischer, Juan Pablo Montero, Andrea Repetto, Klaus Schmidt-Hebbel y dos árbitros anónimos; no obstante, los autores son los únicos responsables del contenido del trabajo.

E-mails: ccontrer@pbct.cl; gedwards@faceapuc.cl; amizala@dii.uchile.cl
} 
nal. Además, con economía, administración y otras disciplinas a nivel internacional. Por otra parte, consideramos los recursos públicos destinados a la investigación en Chile, diferenciando por disciplinas y contrastándolos con un indicador de productividad relativa.

Se comparan dos décadas (1984-2003) de productividad científica medida por publicaciones en revistas indexadas por el Institute for Scientific Information (ISI). Se incluye en el análisis a otros países latinoamericanos que muestran una productividad científica relevante como Argentina, Brasil y México y también a la región latinoamericana en su conjunto. ${ }^{1}$ Asimismo, consideramos en esta comparación a EE.UU., Canadá, Irlanda, Nueva Zelandia y el mundo en su conjunto.

Los indicadores de productividad que utilizamos son el número de artículos por disciplina, las citas correspondientes a esos artículos, el impacto de los artículos, su impacto relativo a la disciplina a nivel mundial y la productividad por peso invertido.

El trabajo concluye que el comportamiento de la producción científica de economía y administración en Chile es similar a la de otros países, en particular, que economía y administración en todos los países publican un menor número de artículos que disciplinas como, por ejemplo, medicina clínica o química. No obstante lo anterior, se aprecia un aumento significativo del número de artículos en economía y administración en la última década, con un impacto mayor al promedio mundial. Es decir, si bien en economía y administración se producen menos artículos que en otras disciplinas, los trabajos publicados tienen un impacto significativo comparado con el promedio mundial de la disciplina. Asimismo, economía y administración muestran uno de los costos más bajos por artículo equivalente, indicando una elevada productividad por peso invertido.

El resto del trabajo está organizado en cuatro secciones. Luego de la introducción en la segunda se compara la productividad científica de las ciencias económicas y administrativas en Chile con la de otros países y con el promedio mundial; esta comparación en cada disciplina con el mundo nos permite contrastar la productividad de economía y administración con otras disciplinas a nivel nacional. En esta parte del trabajo se revisan brevemente otros estudios realizados en esta área, se describen y explican los datos e indicadores utilizados en el estudio y se analizan los resultados de las comparaciones. En la tercera sección se indaga la relación entre recursos asignados por el estado y productividad científica por disciplina en Chile. La cuarta sección presenta las principales conclusiones que surgen del estudio.

\footnotetext{
${ }^{1}$ Koljatic y Silva (2001) muestran que sólo estos países de América Latina tienen una producción científica sustancial en economía y administración.
} 


\section{Comparación de la Productividad Científica de Economía y ADMINISTRACIÓN}

\subsection{Revisión de la literatura}

La investigación acerca de la productividad científica en disciplinas como economía y administración es relativamente escasa. No obstante, existen algunos artículos que jerarquizan departamentos de economía de universidades europeas o norteamericanas, o priorizan revistas del área. ${ }^{2}$

Son escasos, asimismo, los estudios que se focalizan en países en desarrollo; de éstos la gran mayoría compara la productividad en ciencias básicas, sin considerar las ciencias sociales. ${ }^{3}$

Uno de los pocos estudios que aborda la productividad científica comparada de las ciencias económicas y administrativas en países latinoamericanos es el realizado por Koljatic y Silva (2001). Ellos utilizan el Social Science Citation Index (SSCI) y analizan el período 1995 a 1999, concentrándose en cuatro países: Argentina, Brasil, Chile y México, los que presentan una producción significativa en la disciplina. El criterio que utilizan para incluir un país en su estudio es que éste debe tener al menos 25 publicaciones en el período analizado. Por otra parte, los autores se focalizan en artículos publicados por investigadores de universidades en América Latina. El estudio concluye que, al corregir por producto interno bruto (PIB), población y número de estudiantes universitarios, Chile muestra los resultados más favorables en la comparación, de acuerdo a indicadores comúnmente utilizados como número de artículos y citas.

Tanto el número como la calidad de las publicaciones en economía y administración en Latinoamérica, incluido Chile, se han incrementado en los últimos años. Esta situación puede ser explicada por diversos factores, tales como la conformación de una masa crítica de investigadores formados en universidades de primer nivel de EE.UU. y Europa; la mayor facilidad para acceder con rapidez, gracias a internet, a las investigaciones que se realizan en otras partes del mundo; el establecimiento de fondos concursables asignados competitivamente de acuerdo a la calidad de los proyectos e investigadores y el establecimiento de una carrera académica en las universidades con mayor tradición, las que asignan gran importancia a las publicaciones internacionales indexadas en sus procesos de evaluación.

\subsection{Indicadores e información utilizada}

La información con que se realiza este estudio ha sido obtenida de Thomson Scientifics ${ }^{\circledR}$ National Science Indicators (NSI) versión estándar para el

\footnotetext{
${ }^{2}$ Ver Davis (1998), Extejt y Smith (1990), Johnson y Podsakoff (1994), Laban y Piette (1994), Macharzina y Oesterle (1994), Medoff (1996), Zinkhan y Leigh (1999).

${ }^{3}$ Ver Krauskopf, Vera y Welljams-Dorof (1995), Lewison, Fawcett-Jones y Kessler (1993), Osareh y Wilson (1997) y Shrum (1997).
} 
período 1981-2003. ${ }^{4}$ Los artículos considerados en esta base de datos corresponden a lo que se denomina producción citable publicada en revistas indexadas por el Institute for Scientific Information (revistas ISI). Esta producción citable incluye trabajos, notas, reseñas y proceedings papers. ${ }^{5}$

Los artículos son asignados en fuente a una determinada disciplina de acuerdo a la revista en que son publicados ${ }^{6}$, puesto que son las revistas, y no los artículos, las que se clasifican en una determinada disciplina. ${ }^{7}$

Es importante mencionar que algunas revistas, y por tanto sus artículos, pueden ser asignadas a más de una disciplina. El Cuadro A1 del anexo muestra que 7.370 revistas sólo se asignan a una disciplina, mientras que 567 se asignan a dos disciplinas, 17 se asignan a tres disciplinas y 1 se asigna a cuatro disciplinas. Esto significa que la suma de las revistas asignadas a las distintas disciplinas, 8.559, es superior al total de revistas, 7.955. Debido a esto, para cada país considerado en el estudio la suma de artículos de todas las disciplinas es alrededor de un $10 \%$ superior al total de artículos efectivos.

Por otra parte, los artículos se asignan, también en la fuente, a los diferentes países dependiendo de la institución de afiliación, no de la nacionalidad del autor. Si el artículo tiene coautores y cada uno está asociado a instituciones de diferentes países, éste se contabiliza como un artículo en cada país y no se divide entre coautores. Finalmente, si hay un único autor del artículo que tiene doble afiliación en instituciones que pertenecen a distintos países, el artículo es contabilizado doblemente, uno por cada país, pero sólo una vez en la clasificación por disciplinas.

Los dos elementos recién mencionados implican que se debe reconocer algún grado de error o sesgo al comparar distintas disciplinas en un mismo país a no ser que se utilice algún mecanismo de normalización; sin embargo, no existiría sesgo al comparar las mismas disciplinas entre los distintos países.

El trabajo considera, en la segunda parte, información proveniente de la Comisión Nacional de Investigación en Ciencia y Tecnología (CONICYT) y de la Iniciativa Científica MILENIO referida al número de proyectos y recursos asignados para investigación en las diversas disciplinas. En particular, los proyectos del Fondo Nacional de Desarrollo Científico y Tecnológico (FONDECYT), Fondo de Areas Prioritarias (FONDAP) y MILENIO en el mismo período

\footnotetext{
${ }^{4}$ Para mayores detalles ver User Documentation National Science Indicators (2004).

${ }^{5} \mathrm{El}$ incluir notas y reseñas no es una opción de los autores, sino del NSI, ya que ellos consideran en sus bases todos los artículos citables, argumentan que esto es una convención en la literatura cienciométrica. La base de datos en todo caso no incluye editoriales, cartas, correcciones y resúmenes (abstracts).

${ }^{6}$ Una excepción a esta regla son los artículos publicados en las revistas Science, Nature y los Proceedings de la American Academy of Science. En este caso, 60\% de los artículos son asignados a diferentes disciplinas, de acuerdo a un algoritmo especialmente diseñado por ellos, y el resto son clasificados como multidisciplinarios.

${ }^{7}$ Existe una clasificación llamada estándar que incluye 24 disciplinas y una llamada de luxe que incluye 105 subdisciplinas. Nosotros trabajamos con 24 disciplinas.
} 
1984-2003. ${ }^{8}$ Las disciplinas con que se trabaja son las mismas 24 incluidas en la base NSI. ${ }^{9}$

Se utiliza, asimismo, información que permite poner en contexto los distintos países analizados. En particular, el PIB per cápita, el gasto en investigación y desarrollo en universidades como porcentaje del PIB, y la tasa de estudiantes matriculados en educación superior.

Los indicadores de productividad que utilizamos son el número de artículos, las citas correspondientes a esos artículos, el impacto de los artículos y su impacto relativo a la disciplina a nivel mundial.

Es necesario detenernos en este punto y explicar en detalle los indicadores utilizados y justificar su elección. A continuación argumentamos, en primer lugar, por qué utilizamos el número de citas hasta el presente de un artículo y el factor de impacto calculado en base a esas citas y, en segundo lugar, por qué utilizamos el índice de impacto relativo a la disciplina para comparar entre ellas.

El número de citas incluye las citas de un artículo desde la fecha en que se publicó hasta el presente, en nuestro caso el año 2003. El factor de impacto acumulado es calculado como el número de citas hasta el presente de artículos publicados en un determinado año, dividido por el número de artículos publicados en dicho año. Este es diferente al factor de impacto que comúnmente se reporta para evaluar la calidad de las revistas, el cual para un año determinado se calcula como el número total de citas recibidas ese año de los artículos publicados en los dos años anteriores, dividido por el número total de artículos publicados por la revista en esos dos años. Si bien el factor de impacto hasta el presente tiende a subestimar el impacto total de algunos artículos, dado que incluye artículos recientes que pueden demorar en madurar, éste parece más adecuado que el factor de impacto de corto plazo utilizado para evaluar las revistas, el que posee un mayor sesgo temporal puesto que es un indicador de corto plazo.

En efecto, hay disciplinas con mayor rezago en la publicación de artículos y, por ello, se tiende a citar documentos de trabajo que aún no han sido publicados, en vez de artículos ISI. Asimismo, hay disciplinas en que los artículos se citan profusamente, pero por un corto período de tiempo, mientras que en otras éstos continúan siendo citados por muchos años. Este último es el caso de matemáticas, ciencias de la computación y economía y administración que tienen un factor de impacto de corto plazo reducido y un factor de impacto de largo plazo más elevado (ver Contreras, Edwards y Mizala, 2006). ${ }^{10}$

El Cuadro 1 muestra asimismo que hay diferencias estructurales en la productividad científica a nivel mundial. ${ }^{11}$ Determinadas disciplinas como quí-

\footnotetext{
${ }^{8}$ Se hace notar que los proyectos FONDAP comienzan el año 1994 y los MILENIO el año 1999.

${ }^{9}$ En el Cuadro A1 del anexo de Contreras, Edwards y Mizala (2005) se presentan las equivalencias entre la clasificación de disciplinas de FONDECYT y de National Science Indicators (NSI).

${ }^{10} \mathrm{El}$ Cuadro A2 del anexo muestra para las 24 disciplinas estimaciones del factor de impacto de corto y de largo plazo.

${ }^{11}$ En la sección siguiente se muestra que estas diferencias en la productividad científica se observan en cada uno de los países considerados en este estudio.
} 
mica, medicina clínica, biología y bioquímica, neurología y neurocirugía producen un mayor número de artículos en revistas ISI y tienden a tener un mayor número de citas por artículo, medido por el factor de impacto acumulado. También tienen un importante número de citas por artículo disciplinas como inmunología, microbiología, biología molecular y genética y ciencias del espacio. Además, hay diferencias significativas en el número de revistas ISI por disciplina.

Asimismo, como se explicó anteriormente, cuando hay coautores de distintos países se contabiliza doblemente en los registros de citas e impactos, uno por cada país; esto podría sesgar positivamente a aquellas disciplinas que usualmente desarrollan trabajo en equipo a nivel internacional. También podría existir un sesgo por el hecho de que ciertas revistas se asignan a más de una disciplina.

\section{CUADRO 1}

PRODUCTIVIDAD CIENTIFICA EN EL MUNDO POR DISCIPLINA 1984-2003. NUMERO DE ARTICULOS, IMPACTO ACUMULADO Y NUMERO DE REVISTAS POR DISCIPLINA

\begin{tabular}{lrrrr}
\hline Disciplina & $\mathrm{N}^{\circ}$ artículos & $(\%)$ & Impacto & $\mathrm{N}^{\circ}$ revistas ISI \\
\hline Ciencias silvoagropecuarias & 318.460 & 2,3 & 6,40 & 226 \\
Biología y bioquímica & 1.042 .195 & 7,6 & 21,22 & 411 \\
Química & 1.733 .138 & 12,6 & 10,24 & 534 \\
Medicina clínica & 2.946 .677 & 21,3 & 13,44 & 1625 \\
Ciencias de la computación & 131.844 & 1,0 & 4,49 & 201 \\
Ecología y ciencias ambientales & 295.153 & 2,1 & 9,92 & 218 \\
Economía y administración & 185.016 & 1,3 & 7,33 & 304 \\
Educación & 50.819 & 0,4 & 3,75 & 109 \\
Ingeniería & 935.128 & 6,8 & 4,70 & 753 \\
Ciencias de la tierra & 334.047 & 2,4 & 11,33 & 301 \\
Inmunología & 216.652 & 1,6 & 28,76 & 89 \\
Derecho & 37.858 & 0,3 & 6,18 & 95 \\
Ciencias de los materiales & 434.057 & 3,1 & 5,84 & 232 \\
Matemáticas & 240.421 & 1,7 & 4,97 & 211 \\
Microbiología & 294.007 & 2,1 & 17,88 & 104 \\
Biología molecular y genética & 344.314 & 2,5 & 33,09 & 189 \\
Multidisciplinaria & 222.413 & 1,6 & 7,61 & 123 \\
Neurología y neurocirugía & 474.801 & 3,4 & 22,99 & 213 \\
Farmacología & 309.768 & 2,2 & 12,44 & 150 \\
Física & 1.469 .311 & 10,6 & 9,80 & 356 \\
Ciencias de las plantas y animales & 836.454 & 6,1 & 8,44 & 649 \\
Sicología y siquiatría & 352.395 & 2,6 & 12,18 & 490 \\
Ciencias sociales, general & 452.118 & 3,3 & 5,37 & 926 \\
Ciencias del espacio & 146.262 & 1,1 & 16,57 & 50 \\
\hline Suma & 13.803 .308 & 100,0 & 12,22 & 8.559 \\
\hline
\end{tabular}

Fuente: National Science Indicators. 
Debido a todas estas diferencias entre las distintas áreas, este trabajo utiliza el indicador de impacto relativo a la disciplina, el que se obtiene dividiendo el impacto de los artículos en la disciplina en el país (o región) por el impacto de la disciplina a nivel mundial. Este indicador reconoce que las disciplinas tienen distintos patrones de publicación y permite, por tanto, normalizar la productividad científica de distintas disciplinas y países, lo que agrega validez a las comparaciones que se realizan en este estudio. Un impacto relativo mayor que uno significa que los artículos de ese país o región tienen un impacto mayor que el promedio mundial en la disciplina.

\subsection{Productividad científica comparada}

En esta sección, en primer lugar, comparamos la productividad científica de economía y administración en Chile vis a vis otros países y otras disciplinas. En segundo lugar, profundizamos en la comparación de la productividad científica de las distintas disciplinas en Chile, analizando su evolución en el tiempo, luego de dividir el período 1984-2003 en quinquenios.

\section{Comparación con países seleccionados}

El Cuadro 2 presenta el número de artículos y la participación de cada disciplina en la suma de artículos de cada país para el período 1984-2003.

A nivel general y en términos del número total de artículos publicados, destaca claramente EE.UU. con una producción de más de 4,5 millones de artículos en el período 1984-2003, lo que equivale a un 36,5\% de la producción mundial. Le sigue Canadá con un 4,8\% de la producción total. Chile representa un $0,22 \%$ del total de artículos publicados. EE.UU. supera al resto de los países en las 24 disciplinas consideradas. Esto se podría explicar, al menos en parte, porque un número significativo de las revistas científicas indexadas por ISI son de origen estadounidense. ${ }^{12}$

Es obvio que estos resultados están directamente relacionados con el tamaño de los países, tanto en términos de su producto interno bruto y su población, como su inversión en investigación y desarrollo (I\&D) en las universidades y la importancia de su sistema universitario, medido por la tasa de matrícula en educación superior (Cuadro 3). EE.UU. y Canadá efectivamente son los países con mayor producto per cápita y que más invierten en I\&D en las universidades: Canadá un 0,59\% de su PIB y EE.UU. un 0,39\% del PIB; le sigue Nueva Zelandia con una inversión del 0,35\% de su PIB. Chile invierte un 0,25\% del PIB, que es más de lo que invierten Argentina, Brasil y México, siendo estos últimos países de mayor tamaño poblacional y con un PIB per cápita relativamente similar.

\footnotetext{
${ }^{12}$ Seglen (1997) ejemplifica este hecho observando que la base de datos del Institute for Scientific Information incluye dos revistas de origen alemán en el área de ciencias sociales, mientras que la base de datos alemana contiene 542 revistas en esta área.
} 


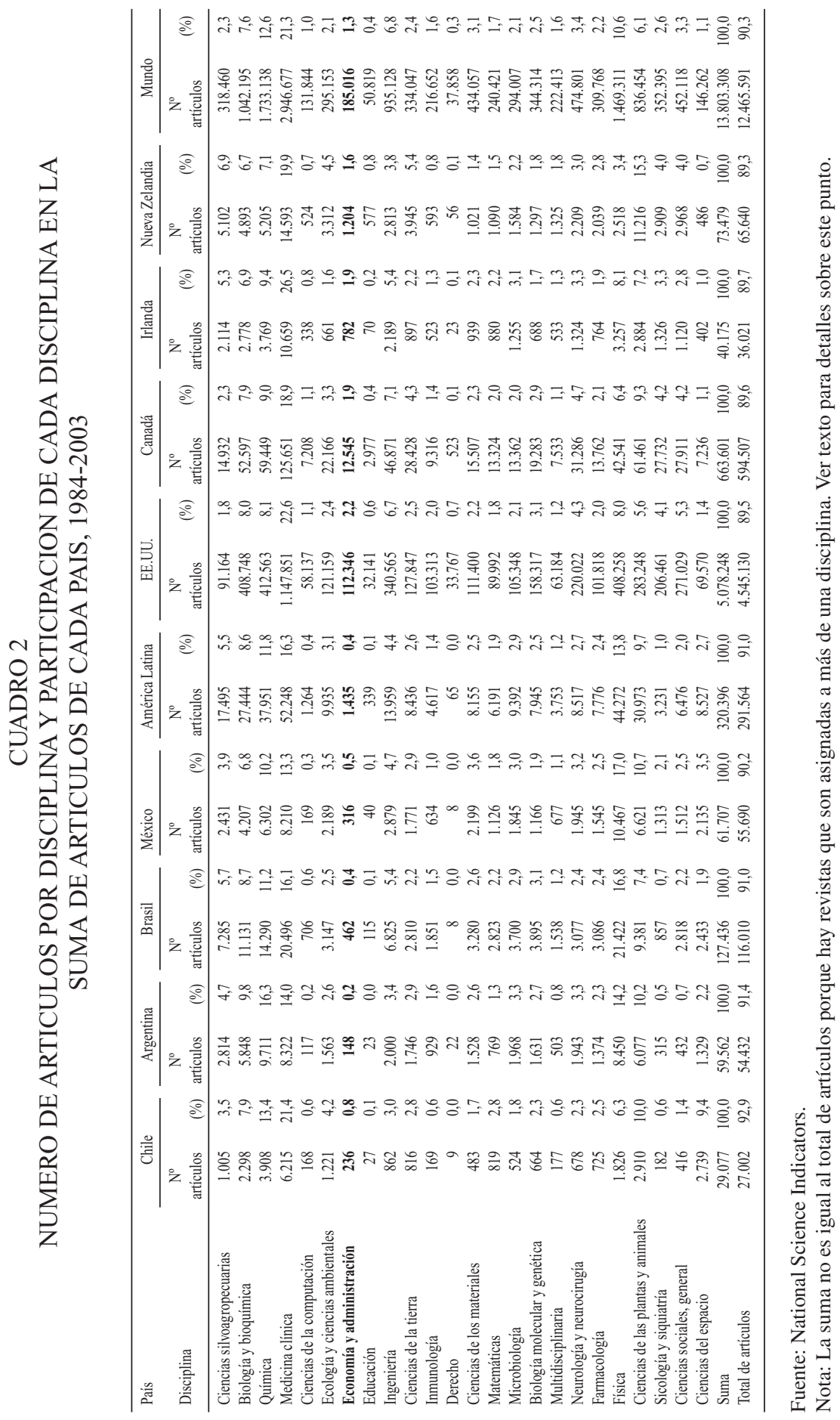




\section{CUADRO 3 \\ NUMERO DE ARTICULOS PUBLICADOS, INVERSION EN INVESTIGACION Y DESARROLLO, PRODUCTO PER CAPITA Y TASA DE MATRICULA EN EDUCACION SUPERIOR. PAISES SELECCIONADOS}

\begin{tabular}{|c|c|c|c|c|c|}
\hline$\square \square \square \square[\operatorname{lis} \square \square \square \square \mathrm{P}$ & $\begin{array}{c}\text { Número total de } \\
\text { artículos publicados } \\
\text { Pal año cada } 1 \text { millón } \\
\text { de habitantes } \\
\text { 1984-2003* }\end{array}$ & $\begin{array}{l}\text { Número de artículos } \\
\text { publicados al año en } \\
\text { Economía y } \\
\text { Administración cada } \\
1 \text { millón de habitantes } \\
\text { 1984-2003* }\end{array}$ & $\begin{array}{c}\text { Inversión en } \\
\text { Investigación y } \\
\text { Desarrollo en las } \\
\text { universidades sobre } \\
\text { PIB } \\
\text { Año 2001 } \\
(\%)\end{array}$ & $\begin{array}{l}\text { PIB per cápita } \\
\text { (precios } \\
\text { constantes: } \\
\text { Laspeyres) } \\
\\
\text { Año } 2000 \\
\text { US\$ de } 1996\end{array}$ & $\begin{array}{c}\text { Tasa de } \\
\text { matrícula en } \\
\text { educación } \\
\text { superior } \\
\text { Año } \\
\text { 2000/2001** } \\
(\%)\end{array}$ \\
\hline Argentina & 96 & 0,3 & 0,15 & 10.995 & 52 \\
\hline Brasil & 45 & 0,2 & 0,15 & 7.185 & 16 \\
\hline Canadá & 1.237 & 26 & 0,59 & 26.922 & 59 \\
\hline Chile & 117 & 1 & 0,25 & 9.920 & 38 \\
\hline Irlanda & 590 & 13 & 0,24 & 26.379 & 47 \\
\hline América Latina & 39 & 0,2 & n.a. & 7.071 & $25 * * *$ \\
\hline México & 38 & 0,2 & 0,12 & 8.766 & 20 \\
\hline Nueva Zelandia & 1.097 & 20 & 0,35 & 18.824 & 69 \\
\hline Estados Unidos & 1.047 & 26 & 0,39 & 33.308 & 71 \\
\hline
\end{tabular}

Fuente: OECD, Main Science and Technology Indicators, noviembre 2005.

* Este indicador es el cuociente entre el número total de artículos en el período y la suma de la población de cada año.

** En cada caso se consideró la información del último año disponible para todos los países.

*** Incluye Argentina, Bolivia, Brasil, Chile, Colombia, Ecuador, Guyana, Paraguay, Perú, Surinam, Uruguay y Venezuela.

Si consideramos el conjunto de las disciplinas, Chile publicó 117 artículos al año por cada millón de habitantes en el período 1984-2003, Argentina 96, Brasil 45 y México 38. Esto contrasta con 1.047 publicados en EE.UU. y 1.237 en Canadá. Países como Irlanda y Nueva Zelandia publicaron 590 y 1.097 artículos anuales cada millón de habitantes, respectivamente (Cuadro 3).

Por su parte, en economía y administración Chile tiene el mejor desempeño de América Latina con 1 artículo al año por cada millón de habitantes en el período 1984-2003, mientras que Brasil y México tienen 0,2 artículos y Argentina 0,3 artículos anuales. Sin embargo, Chile está lejos de países desarrollados como EE.UU. y Canadá, que publicaron 26 artículos anuales por cada millón de habitantes en el mismo período, y de países como Nueva Zelandia e Irlanda que publicaron 20 y 13 artículos, respectivamente. Como ya mencionamos, todas estas cifras están altamente correlacionadas con el PIB per cápita, la inversión en I\&D a nivel universitario y la tasa de matrícula en educación superior (Cuadro $\square 3$ ).

Volviendo al Cuadro 2 podemos observar que la composición disciplinaria de la producción científica es en general similar en todos los países. Medicina clínica es la disciplina que más artículos indexados produce; su participación fluctúa entre un 13,3\% en México y un 26,5\% en Irlanda. Sólo 
en México y Argentina no es la disciplina con más alta participación porque es superada por física y por química, respectivamente. Por otra parte, la disciplina con menor producción científica es derecho, ya que su participación fluctúa entre prácticamente $0 \%$ en todos los países latinoamericanos considerados y $0,7 \%$ en EE.UU.

Los países de América Latina tienden a tener una mayor participación relativa que el resto en química, física y ciencias de las plantas y animales, y una menor participación relativa en disciplinas como ciencias sociales, sicología y siquiatría.

En economía y administración también se observan diferencias entre los países latinoamericanos y el resto de los países considerados. Mientras que en Latinoamérica la participación más alta la tiene Chile con un 0,8\%, en EE.UU. la participación es 2,2\%, en Canadá e Irlanda un 1,9\% y en Nueva Zelandia un $1,6 \%$. No obstante, en todos los países la producción científica de economía y administración es significativamente menor que en las ciencias básicas como química, física, biología y bioquímica y que en otras disciplinas como ingeniería, ciencias de las plantas y animales y medicina clínica.

Al comparar la estructura de la producción científica chilena con la de otros países destacan las ciencias del espacio (astronomía) con una producción equivalente al 9,4\% del total. Esto se explica en gran medida por la ubicación en el norte de Chile de los observatorios astronómicos.

Pero no sólo interesa el número de publicaciones, sino también su calidad, medida por el número de citas. El Cuadro 4 presenta dos indicadores de impacto de los artículos publicados en el período 1984-2003, el impacto acumulado hasta el presente y el impacto relativo a la disciplina a nivel mundial.

Lo primero que se observa es que algunas disciplinas, independientemente de los países donde se produzcan los artículos, tienen un mayor impacto, es decir, tienden a citarse más. Este es el caso de biología y bioquímica, inmunología, microbiología, biología molecular y genética, neurología y neurocirugía y ciencias del espacio. Por esta razón, como ya argumentamos, lo que importa para medir la calidad de la producción científica es el impacto relativo a la disciplina a nivel mundial.

Si observamos el impacto relativo a la disciplina podemos concluir que de los países analizados, EE.UU. tiene un impacto superior al promedio mundial en todas las disciplinas, le sigue Canadá con un alto número de disciplinas (19) con impacto relativo superior a 1; más lejos se encuentran Nueva Zelandia (6 disciplinas) e Irlanda (3). En América Latina en su conjunto, y en Argentina, Brasil y México por separado, ninguna disciplina tiene un impacto superior al promedio mundial. Chile es el único país que tiene tres disciplinas con un impacto igual o superior a 1 (economía y administración, ciencias del espacio y el área multidisciplinaria). 


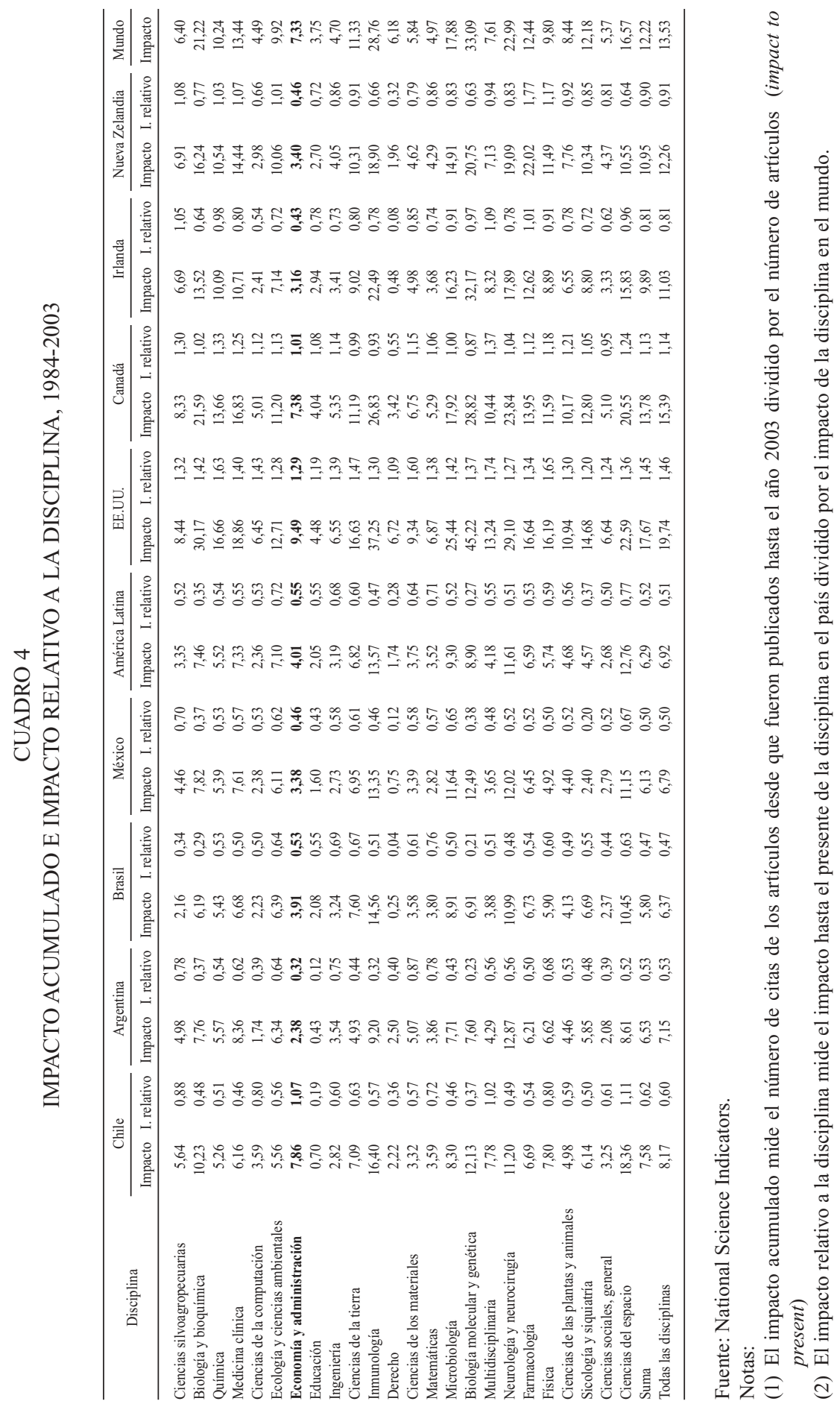




\section{Productividad por disciplina en Chile}

La información para Chile está en los cuadros 2 y 4 antes mencionados. En el Cuadro 2 se puede observar el número de artículos publicados en cada disciplina en el período 1984-2003, así como la participación de las disciplinas en la producción científica nacional. Por su parte, en el Cuadro 4 se encuentra el impacto acumulado hasta el presente y el impacto relativo a la disciplina a nivel mundial.

En términos del número de artículos destaca en primer lugar medicina clínica, con un 21,4\% de los artículos publicados en el país en el período 1984-2003, le siguen química con un $13,4 \%$, ciencias de las plantas y los animales $10 \%$, ciencias del espacio $9,4 \%$ y biología y bioquímica $7,9 \%$, economía y administración representa sólo el $0,8 \%$ de los artículos publicados. Como ya comentamos, la participación de las disciplinas en el número de artículos es, en general, consistente con lo que ocurre en los distintos países analizados y a nivel mundial.

La calidad de los artículos medida a través del impacto acumulado al presente muestra que en Chile la disciplina con mayor impacto es ciencias del espacio con un factor de 18, seguida por inmunología con 16,4, biología molecular y genética con 12,1 , neurología y neurocirugía con 11,2 y biología y bioquímica con 10,2. Los artículos chilenos de economía y administración tienen un impacto acumulado de 7,9.

Ahora bien, si analizamos lo que ocurre con el impacto relativo a la disciplina, que permite normalizar la productividad científica de diversas áreas, Chile, como ya se mencionó, posee sólo 3 disciplinas con un impacto relativo superior a 1: economía y administración con 1,07, ciencias del espacio 1,11 y el área multidisciplinaria 1,02. Recordemos que un impacto relativo superior a 1 significa que los artículos chilenos en estas disciplinas tienen un impacto mayor que el promedio mundial (Gráfico 1).

Para dar cuenta de la evolución en el tiempo de la productividad científica por disciplina, el Cuadro 5 muestra la evolución del número de artículos y el impacto relativo a la disciplina en períodos de 5 años, desde el año 1984 al 2003. Se observa un número creciente de artículos a través del período. De hecho, son pocas las disciplinas donde no ha habido un aumento sustantivo de publicaciones, entre ellas, derecho, educación, y sicología y siquiatría. Este comportamiento es similar al que han tenido las distintas disciplinas en el resto del mundo. ${ }^{13}$ Es interesante sí puntualizar el notable aumento de los artículos indexados en economía y administración; entre 1984 y 1988 se escribieron 15 artículos, cifra que aumentó a 106 artículos en el quinquenio 1999-2003.

En términos de la evolución del impacto relativo a la disciplina se mantiene lo observado anteriormente en Chile: las tres disciplinas ya mencionadas son

\footnotetext{
${ }^{13}$ En el Cuadro A4 de Contreras, Edwards y Mizala (2005) se presenta la evolución del número de artículos y el impacto relativo de cada una de las disciplinas para todos los países que se han considerado en el estudio.
} 


\section{GRAFICO 1 \\ IMPACTO RELATIVO POR DISCIPLINA \\ CHILE, 1984-2003}

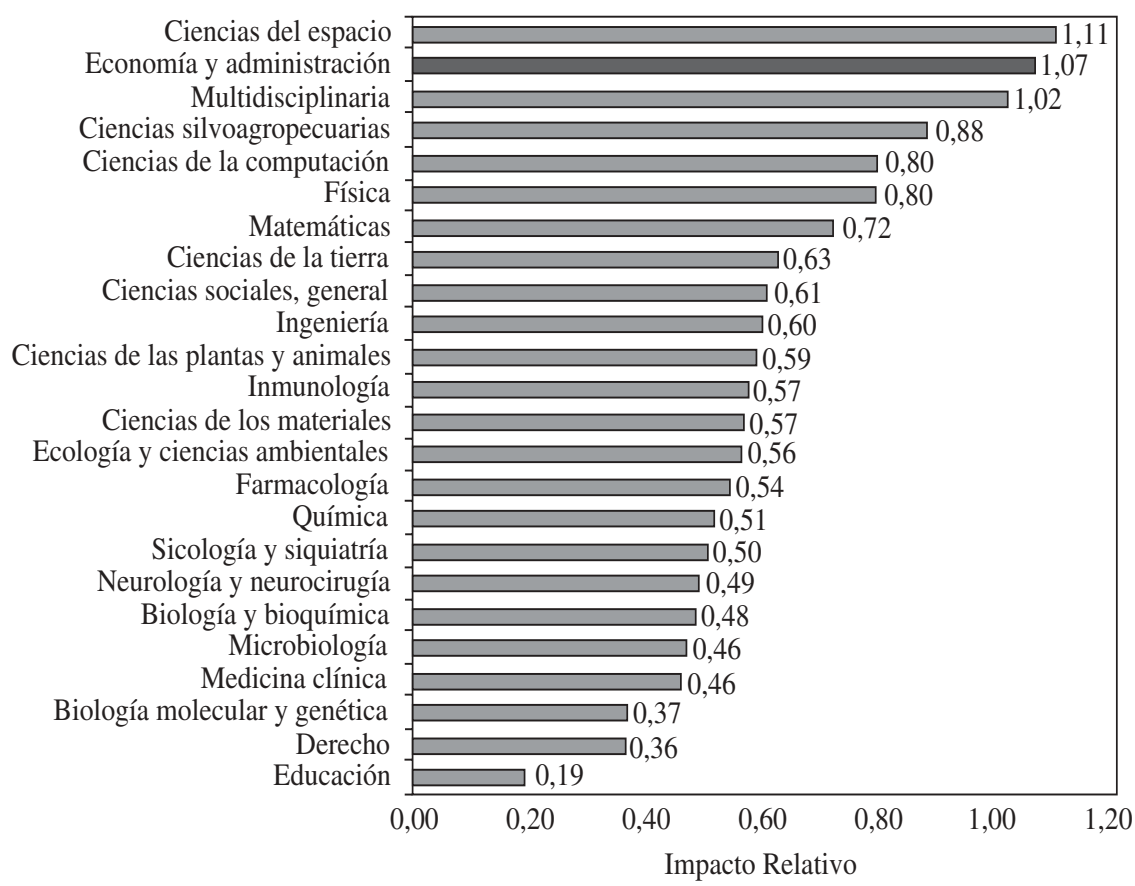

Fuente: Cuadro 4.

las que tienen, en general, el mayor impacto relativo en cada uno de los quinquenios. En el caso de economía y administración el impacto acumulado de los artículos publicados en el quinquenio 1984-88 es muy elevado, 5,06, lo que se explica fundamentalmente por un artículo en particular. ${ }^{14}$ Sin embargo, en el período 1994-98 y en el 1999-03, los artículos publicados en esta disciplina también tienen un impacto relativo igual o mayor al promedio mundial de la disciplina, 1,02 y 1,10 , respectivamente. Por lo tanto, se observa que ha aumentado la producción de artículos indexados en economía y administración, los cuales han presentado un impacto relevante comparado con el desempeño de la disciplina a nivel mundial.

\footnotetext{
${ }^{14}$ Este mismo fenómeno podría estar ocurriendo en otras disciplinas, sin embargo, este punto no fue examinado en este trabajo.
} 


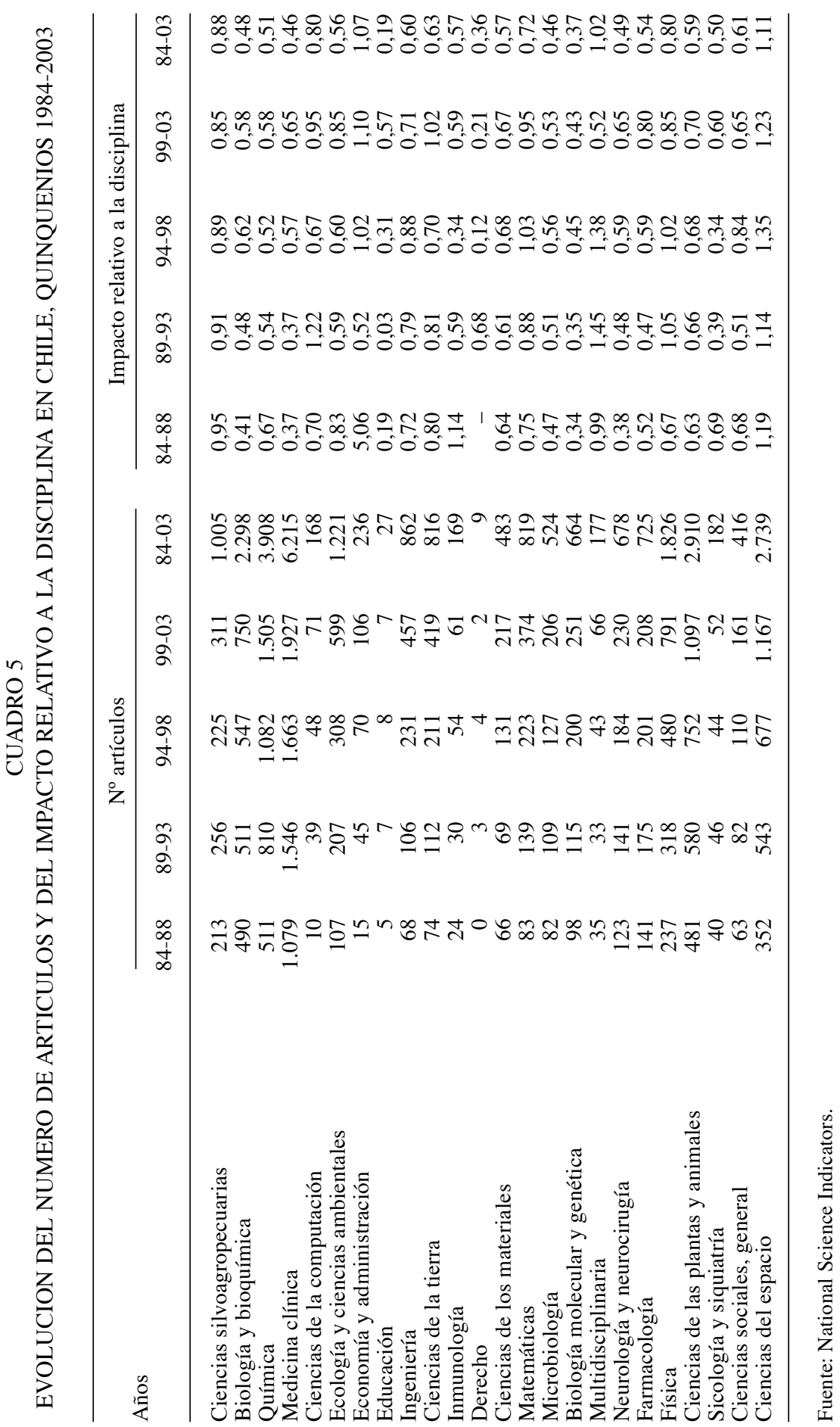




\section{Recursos Públicos Para InVestigación Asignados POR DisCIPLINAS}

En esta parte del trabajo contrastamos el monto de recursos públicos destinados a financiar investigación científica, publicable en revistas de prestigio internacional, en las distintas disciplinas con su eficiencia relativa. La eficiencia de cada disciplina la medimos en términos de la producción de trabajos científicos y su calidad; en particular, a través del número de artículos publicados en revistas ISI y las citas que esos trabajos reciben, comparando esta producción con el desempeño de la disciplina a nivel mundial.

Es importante notar que los recursos considerados en este análisis se refieren sólo a aquellos destinados a financiar investigación científica y no transferencia tecnológica o investigación en políticas públicas. En estos casos el indicador de productividad de la inversión sería diferente, puesto que la contribución al país se daría, por ejemplo, a través de un mayor desarrollo tecnológico o una mayor disponibilidad de opinión experta en temas prácticos.

El Cuadro 6 presenta los fondos públicos asignados a cada disciplina por FONDECYT en el período 1984-2003 y en cada uno de los quinquenios. El Cuadro 7 contrasta los recursos y proyectos asignados por FONDECYT en el quinquenio 1999-2003 con el número de investigadores en cada disciplina. El Cuadro 8 presenta los proyectos FONDAP y MILENIO. ${ }^{15}$

Los recursos de FONDECYT adjudicados en el período 1984-2003 están concentrados en unas pocas áreas, fundamentalmente biología y bioquímica, química, medicina clínica e ingeniería. Estas cuatro disciplinas han recibido el $51 \%$ de los recursos asignados por FONDECYT. Es importante destacar que no se trata sólo de los recursos -ya que el monto de éstos puede estar asociado a diferencias en las necesidades de cada disciplina, por ejemplo, compra de equipos caros, instalación de laboratorios, etc.- sino que obtienen también un mayor número de proyectos; estas cuatro disciplinas concentran el $47 \%$ de los proyectos del período.

Esta concentración de recursos públicos y proyectos parece no explicarse por la existencia de un mayor número de investigadores en estas disciplinas, entendiendo por investigadores a aquellos que han concursado a proyectos FONDECYT en el período 1999-2003. Sólo medicina clínica tiene una mayor participación en el total de investigadores que de proyectos (Cuadro 7, Gráfico 2). ${ }^{16}$

Por su parte, los proyectos FONDAP y MILENIO también han financiado con recursos significativos a biología y bioquímica. En el período 1994-2003 esta disciplina ha recibido $\$ \square 6.713$ millones de junio del 2004lo que equivale a

\footnotetext{
${ }^{15}$ El Cuadro 7 se focaliza en los proyectos FONDECYT del quinquenio 1999-2003 ya que contrastamos los proyectos y los recursos asignados con el número de investigadores que han concursado a proyectos FONDECYT en ese período.

${ }^{16}$ Este análisis no corrige por el número de investigadores desalentados en las distintas disciplinas, que dada la baja probabilidad de obtener financiamiento de FONDECYT, podrían decidir no postular a estos recursos.
} 


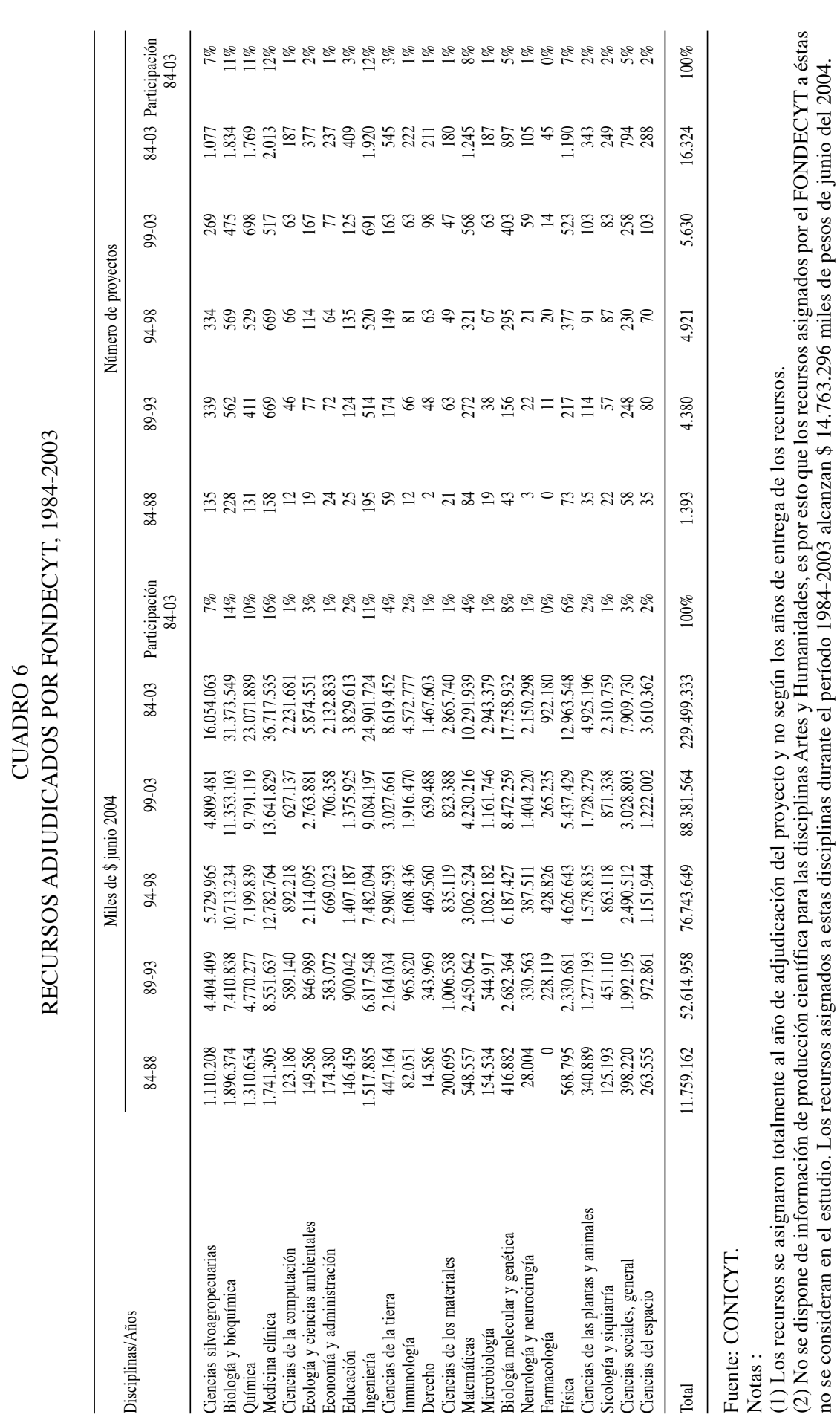




\section{CUADRO 7}

RECURSOS ADJUDICADOS POR FONDECYT Y NUMERO DE INVESTIGADORES POR DISCIPLINA, 1999-2003

\begin{tabular}{|c|c|c|c|c|c|c|}
\hline \multirow{2}{*}{ Disciplinas/Años } & \multicolumn{2}{|c|}{ Miles de $\$$ junio 2004} & \multicolumn{2}{|c|}{$\mathrm{N}^{\mathrm{o}}$ proyectos } & \multicolumn{2}{|c|}{ Investigadores } \\
\hline & $99-03$ & $(\%)$ & $99-03$ & $(\%)$ & $\mathrm{N}^{\circ}$ & $(\%)$ \\
\hline Ciencias silvoagropecuarias & 4.809 .481 & 5 & 269 & 5 & 450 & 7 \\
\hline Biología y bioquímica & 11.353 .103 & 13 & 475 & 8 & 405 & 7 \\
\hline Química & 9.791 .119 & 11 & 698 & 12 & 468 & 8 \\
\hline Medicina clínica & 13.641 .829 & 15 & 517 & 9 & 1.135 & 18 \\
\hline Ciencias de la computación & 627.137 & 1 & 63 & 1 & 67 & 1 \\
\hline Ecología y ciencias ambientales & 2.763 .881 & 3 & 167 & 3 & 128 & 2 \\
\hline Economía y administración & 706.358 & 1 & 77 & 1 & 226 & 4 \\
\hline Educación & 1.375 .925 & 2 & 125 & 2 & 410 & 7 \\
\hline Ingeniería & 9.084 .197 & 10 & 691 & 12 & 552 & 9 \\
\hline Ciencias de la tierra & 3.027 .661 & 3 & 163 & 3 & 188 & 3 \\
\hline Inmunología & 1.916 .470 & 2 & 63 & 1 & 61 & 1 \\
\hline Derecho & 639.488 & 1 & 98 & 2 & 150 & 2 \\
\hline Ciencias de los materiales & 823.388 & 1 & 47 & 1 & 69 & 1 \\
\hline Matemáticas & 4.230 .216 & 5 & 568 & 10 & 205 & 3 \\
\hline Microbiología & 1.161 .746 & 1 & 63 & 1 & 59 & 1 \\
\hline Biología molecular y genética & 8.472 .259 & 10 & 403 & 7 & 231 & 4 \\
\hline Neurología y neurocirugía & 1.404 .220 & 2 & 59 & 1 & 71 & 1 \\
\hline Farmacología & 265.235 & 0 & 14 & 0 & 25 & 0 \\
\hline Física & 5.437 .429 & 6 & 523 & 9 & 209 & 3 \\
\hline Ciencias de las plantas y animales & 1.728 .279 & 2 & 103 & 2 & 174 & 3 \\
\hline Sicología y siquiatría & 871.338 & 1 & 83 & 1 & 208 & 3 \\
\hline Ciencias sociales, general & 3.028 .803 & 3 & 258 & 5 & 623 & 10 \\
\hline Ciencias del espacio & 1.222 .002 & 1 & 103 & 2 & 53 & 1 \\
\hline Total & 88.381 .564 & 100 & 5.630 & 100 & 6.167 & 100 \\
\hline
\end{tabular}

Fuente: CONICYT.

Notas: ver notas Cuadro 6.

Se considera como investigador, a aquellos que han postulado al FONDECYT durante los años 2000-2003.

GRAFICO 2

RECURSOS FONDECYT VS NUMERO DE INVESTIGADORES POR DISCIPLINA (PARTICIPACION)

1999-2003

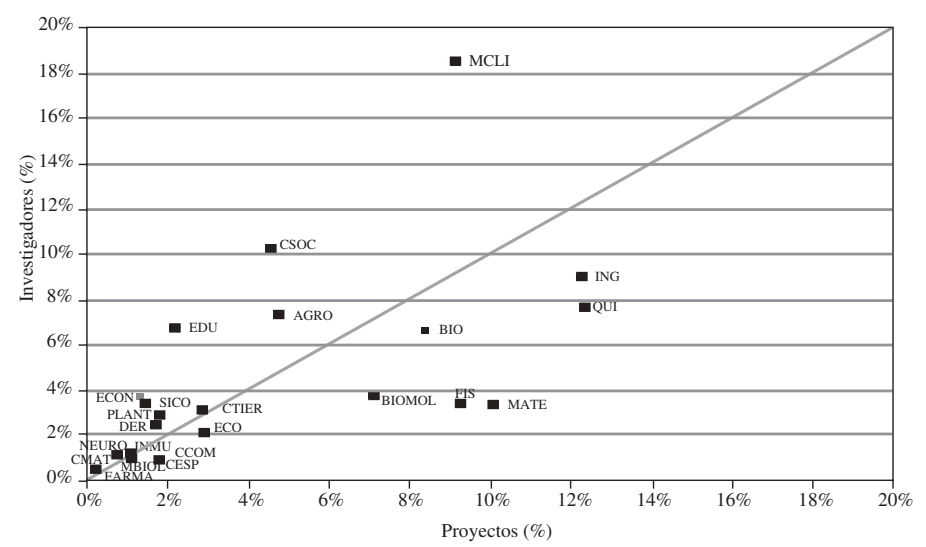

Fuente: Cuadro 7. 
un $29 \%$ del total de recursos asignados para proyectos FONDAP. Además, ella ha obtenido \$ $\square 9.779$ millones del Pograma MILENIO, lo que equivale al 53\% del total asignado por esta iniciativa (Cuadro 8). ${ }^{17}$

Otras disciplinas que han recibido un fuerte apoyo financiero de los proyectos FONDAP son ecología y ciencias ambientales, ingeniería, ciencias de la tierra, matemáticas y ciencias del espacio. Estas disciplinas, excepto la última, también han sido financiadas por la iniciativa científica MILENIO (Cuadro 8). ${ }^{18}$

Es razonable preguntarse qué justifica el grado de concentración de los recursos públicos para investigación en el país en estas disciplinas específicas. Más aún dado que su participación en el número de artículos indexados es bastante menor que su participación en los recursos asignados y, más importante aún, su impacto es menor que el promedio mundial de la disciplina (Cuadros $\square 2, \square 4 \square$ y $\square$ ).

En el Cuadro 9 se presenta un indicador de la relación entre los recursos asignados y la productividad científica de las distintas disciplinas. Se incluye el total de recursos públicos para investigación obtenido por cada disciplina en el período 1984-2003, el número de artículos equivalentes, y los recursos por artículo equivalente. El número de artículos equivalentes se obtiene multiplicando el número de artículos por el impacto relativo a la disciplina a nivel mundial y permite normalizar el número de artículos.

Es necesario hacer notar que el indicador pesos por artículo equivalente no toma en cuenta el hecho de que en determinadas disciplinas se publica normalmente un mayor número de artículos por investigador. En efecto, en algunas disciplinas es frecuente observar investigadores que publican un elevado número de artículos por año, algo que es absolutamente impensado en otras. Por ejemplo, en economía, James Heckman de la U. de Chicago, Peter Phillips, de la U. de Yale, y Jean Tirole de la Universidad de Toulouse, que de acuerdo a Coupé (2003) están entre los investigadores más productivos en el área, publicaron 88 , 71 y 49 artículos, respectivamente, en el período 1995-2004. En contraste, uno de los investigadores más fructíferos en química, John Huffman, tiene 294 publicaciones en el mismo período, mientras que en ciencias de los materiales uno de los más productivos es Jeffery Dahn, con 186 publicaciones en el período. ${ }^{19}$

Tres disciplinas, ciencias del espacio, ciencias de las plantas y animales y farmacología, tienen una mayor eficiencia científica, en términos de recursos por artículo equivalente, que economía y administración. El resto de las disciplinas presentan una menor eficiencia que economía y administración, es decir, tienen un mayor costo por artículo equivalente.

\footnotetext{
${ }^{17}$ Los proyectos FONDAP comenzaron en 1994 y la Iniciativa Científica MILENIO en 1999.

${ }^{18}$ Un 26,5\% de los recursos asignados por la iniciativa científica MILENIO se han orientado a química, ciencias de la computación, biología molecular y genética, neurología y neurocirugía y física.

${ }^{19}$ Los cinco investigadores mencionados aparecen en la lista de Highly cited authors del ISI. Sin embargo, podría haber otros investigadores en otras disciplinas con un mayor número de artículos que Huffman y Dahn.
} 


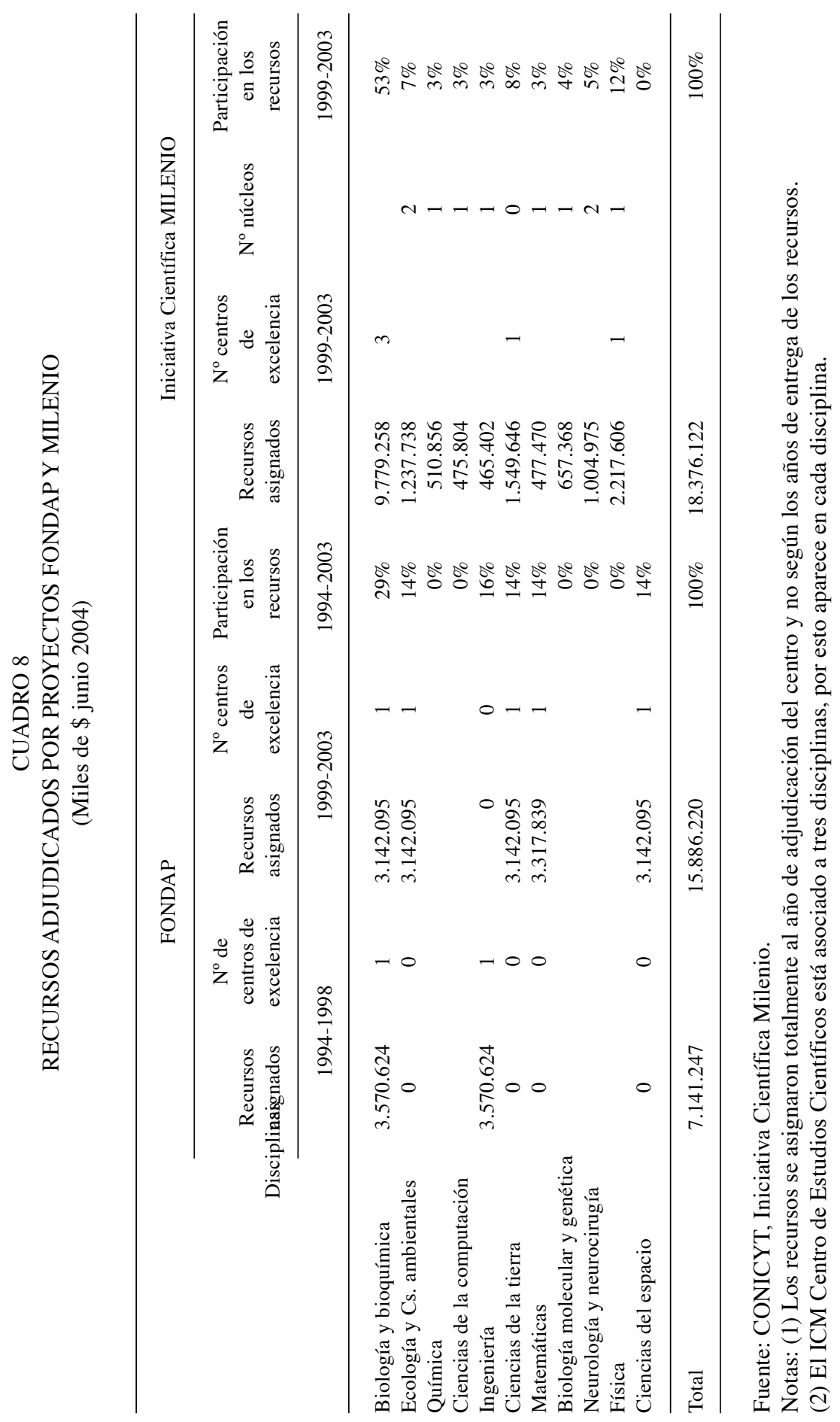




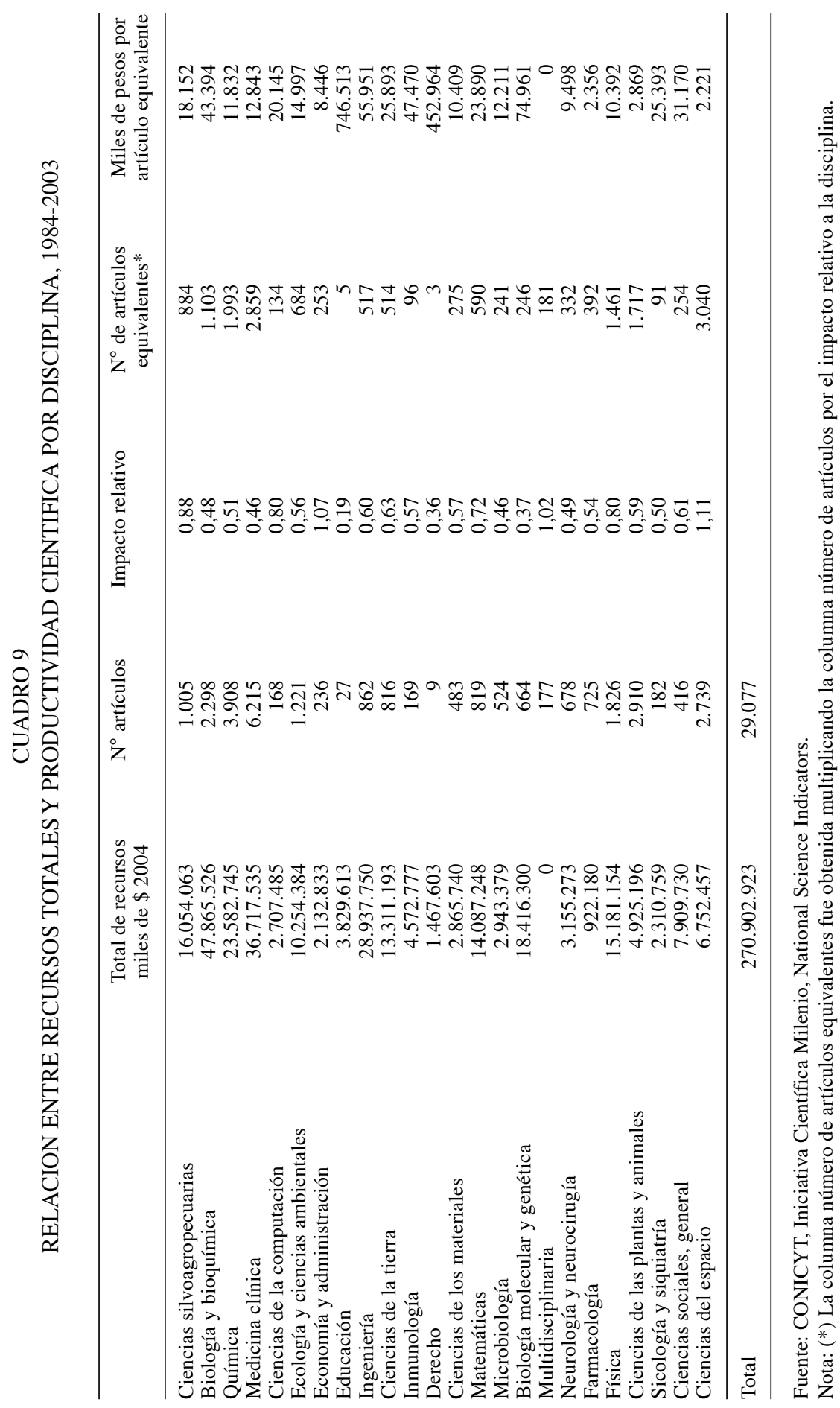




\section{Conclusiones}

Los datos analizados nos permiten concluir que el comportamiento de la productividad científica por disciplina en Chile es similar al de otros países, esto es, hay disciplinas en las que sistemáticamente se produce un mayor número de artículos y que tienden a citarse en mayor medida que el resto.

Chile muestra un desempeño mejor que otros países de América Latina en su producción de artículos científicos, una vez que se corrige por su tamaño. Sin embargo, muestra un claro rezago al compararse con países desarrollados como EE.UU. y Canadá y con países emergentes como Irlanda y Nueva Zelandia.

Se observa una fuerte concentración de los fondos públicos orientados a financiar la investigación científica en Chile en unas pocas disciplinas, principalmente biología y bioquímica, ingeniería, medicina clínica y química. Los datos respecto del número de investigadores activos de estas disciplinas, su participación en la productividad científica nacional y su impacto relativo a nivel mundial no parecen justificar la asignación preferencial de estos recursos.

Economía y administración tienen una baja participación en la producción científica nacional. Si comparamos con las ciencias básicas, en economía y administración se publica un menor número de artículos; sin embargo, este comportamiento es similar a lo que ocurre en otros países. No obstante, es interesante destacar que se observa un aumento significativo del número de artículos en la última década con un impacto mayor al promedio mundial, es decir, si bien en economía y administración se publica menos que en otras disciplinas, los artículos publicados tienen un impacto significativo comparado con el promedio mundial de la disciplina.

Finalmente, si consideramos los recursos públicos para investigación asignados a economía y administración en el período estudiado, se concluye que ésta es una de las disciplinas que revela uno de los costos más bajos por artículo equivalente, mostrando una elevada productividad por peso invertido.

\section{REFERENCIAS}

Contreras, C., Edwards, G. y A. Mizala (2005). "La productividad científica de economía y administración en Chile. Un análisis comparativo", Dcto. de Trabajo $\mathrm{N}^{\circ} 213$ Centro de Economía Aplicada, Depto. Ingeniería Industrial, U. de Chile, y $\mathrm{N}^{\circ} 301$ Instituto de Economía de la Pontificia U. Tatólica de Chile.

Contreras, C., Edwards, G. y A. Mizala (2006). "The Current Impact Factor and the Long-Term Impact of Scientific Journals by Discipline: A Logistic Diffusion Model Estimation", Scientometrics (por aparecer). 
Coupé, T. (2003). "Revealed Performances: Worldwide Rankings of Economists and Economics Departments, 1990-2000", Journal of the European Economic Association, 1(6): 1309-1345.

Davis, J. B. (1998). "Problems in Using the Social Sciences Citation Index to Rank Economics Journals", American Economist, 42 (2): 59-64.

Extejt M. M. y J. E. Smith (1990). “The Behavioral Sciences and Management: An Evaluation of Relevant Journals", Journal of Management, 16: 259-551

Johnson J.L. y P. M. Podsakoff (1994). "Journal Influence in the Field of Management: An Analysis Using Salancik's Index in a Dependency Network", Academy of Management Journal, 37: 1392-1407.

Koljatic, M. y M. Silva (2001). "The International Publication Productivity of Latin American Countries in the Economics and Business Administration Fields", Scientometrics 51 (2): 381-394.

Krauskopf, M., Vera, M. I. y A. Welljams-Dorof (1995). "A Citationist Perspective on Science in Latin America and the Caribbean, 1981-1993", Scientometrics 34 (1): 3-25.

Laban, D. N. y M. J. Piette (1994). "The Relative Impacts of Economics Journals: 19701990, Journal of Economic Literature, 32: 640-666.

Lewison, G., Fawcett-Jones, A. y C. Kessler (1993). "Latin American Scientific Output 1986-91 and International co-authorship patterns", Scientometrics 27: 317336.

Macharzina, K. y M. J. Oesterle (1994). "International Comparative Evaluation of North American and German Research Output in Business and Management", Management International Review, 34: 255-266.

Medoff , M. H. (1996). “A Citation-Based Analysis of Economists and Economic Programs", American Economist, 40:46-54.

Osareh, F. y C. Wilson (1997). "Third World Countries (WC) Research Publications by Disciplines: A country-by-Country Citation Analysis", Scientometrics 39口(3):253-266.

Shrum, W. (1997). "View from afar: 'Visible' Productivity of Scientists in Developing World”, Scientometrics 40: 215-235.

Seglen, P. O. (1997). "Why the Impact Factor of Journals Should Not Be Used for valuating Research". BMJ: 314-497.

Zinkhan, G. M. y T. W. Leigh (1999). "Assessing the Quality Ranking of the Journal of Advertising, 1986-1997”, Journal of Advertising, 28 (2): 51-70. 


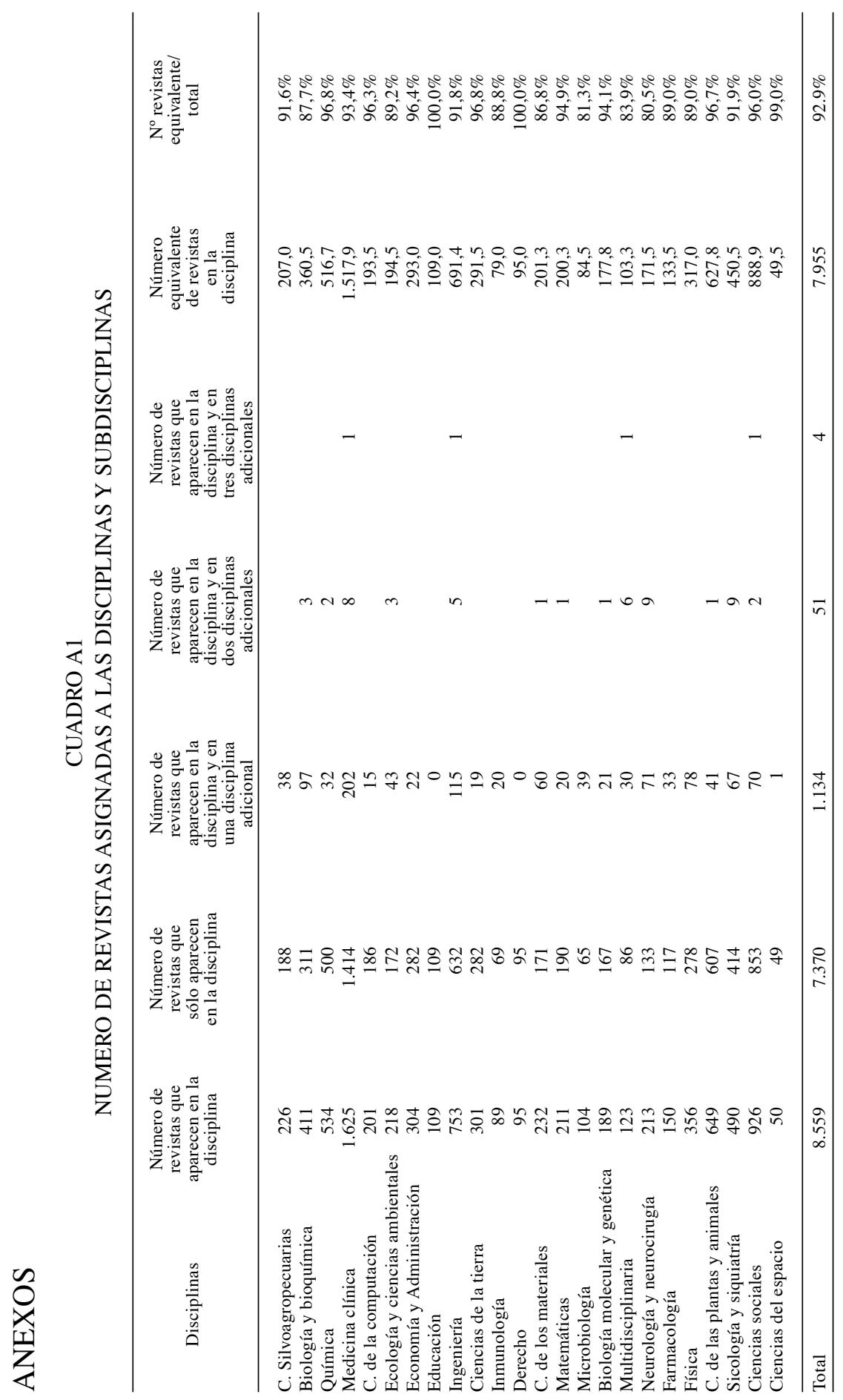




\section{CUADRO A2 \\ FACTOR DE IMPACTO DE CORTO Y LARGO PLAZO POR DISCIPLINA}

\begin{tabular}{lccr}
\hline & $\begin{array}{c}\text { Factor de } \\
\text { impacto de } \\
\text { largo plazo }\end{array}$ & $\begin{array}{c}\text { Factor de } \\
\text { impacto de } \\
\text { corto plazo } \\
\text { Disciplina }\end{array}$ & M/CIF \\
& M & 0,66 & \\
\hline Ciencias silvoagropecuarias & 8,49 & 1,94 & 12,90 \\
Biología y bioquímica & 29,40 & 0,90 & 15,15 \\
Química & 15,52 & 1,37 & 17,29 \\
Medicina clínica & 18,82 & 0,25 & 13,69 \\
Ciencias de la computación & 14,13 & 0,88 & 55,82 \\
Ecología y ciencias ambientales & 15,81 & 0,48 & 18,05 \\
Economía y administración & 11,78 & 0,27 & 24,49 \\
Educación & 5,14 & 0,39 & 19,07 \\
Ingeniería & 7,55 & 0,89 & 19,60 \\
Ciencias de la tierra & 18,66 & 2,57 & 20,86 \\
Inmunología & 41,39 & 0,73 & 16,13 \\
Derecho & 7,26 & 0,57 & 9,91 \\
Ciencias de los materiales & 8,77 & 0,30 & 15,29 \\
Matemáticas & 10,28 & 1,85 & 34,43 \\
Microbiología & 23,93 & 3,32 & 12,96 \\
Biología molecular y genética & 47,05 & 0,97 & 14,17 \\
Multidisciplinaria & 9,59 & 1,92 & 9,86 \\
Neurología y neurocirugía & 35,67 & 1,14 & 18,55 \\
Farmacología & 17,31 & 0,75 & 15,19 \\
Física & 16,43 & 0,75 & 21,84 \\
Ciencias de las plantas y los animales & 11,90 & 0,84 & 15,83 \\
Sicología y siquiatría & 18,92 & 0,48 & 22,57 \\
Ciencias sociales, general & 7,52 & 1,38 & 15,60 \\
Ciencias del espacio & 26,68 & & 19,32 \\
\hline & & &
\end{tabular}

Fuente: Contreras, Edwards y Mizala (2006).

Nota: El impacto de largo plazo fue estimado usando un modelo de difusión logístico, relacionando los resultados al factor de impacto de corto plazo (current impact factor). 